\title{
Comparison of maximal achievable contrast in scalar, Stokes, and Mueller images
}

\author{
François Goudail \\ Laboratoire Charles Fabry de l'Institut d'Optique, CNRS, Université Paris-Sud, Campus Polytechnique, \\ RD 128, 91127 Palaiseau, France (francois.goudail@institutoptique.fr)
}

Received May 17, 2010; revised July 6, 2010; accepted July 6, 2010;

posted July 13, 2010 (Doc. ID 128602); published July 27, 2010

\begin{abstract}
We compare the performances of three different polarimetric imaging modalities, scalar, Stokes, and Mueller, in terms of achievable contrast for target detection applications. These modalities require, respectively, 1, 4, and 16 intensity measurements to form the polarimetric image. We show that the technique that leads to the best contrast is the scalar one, which requires only one optimized measurement. (c) 2010 Optical Society of America OCIS codes: $260.5430,030.4280$.
\end{abstract}

Polarization images are measures of some characteristics of the polarization state of the light scattered by a scene. They can reveal contrasts that do not appear in classical intensity images and find many applications in remote sensing, biomedical imaging, or industrial control. Cost, size, and technological complexity of polarimetric imagers depend on the number of polarimetric parameters they measure. In this context, a key issue is to evaluate the added value of each measured polarimetric parameter in order to optimize the compromise between complexity and efficiency of these systems. In target detection applications, the relevant criterion for quantifying the performance of an imaging configuration is contrast (or discrimination ability) between a target region $a$ and a background region $b$. Analysis of the contrast and its optimization in polarimetric images have been investigated in the radar [1] and optics [2-4] communities. In this Letter, our purpose will be to determine and compare the best achievable contrast in three different polarization imaging modalities: scalar, Stokes, and Mueller. The obtained conclusions will have important applications in domains where optimizing the contrast is a key issue, such as in remote sensing or biomedical imaging.

We use the Mueller formalism to represent the polarimetric properties of the scene, which is assumed to be composed of two regions: a target with Mueller matrix $M_{a}$ and a background with Mueller matrix $M_{b}$ (in this Letter, capital letters represent matrices, bold letters represent vectors, and normal letters represent scalar numbers). The scene is illuminated with purely polarized light that can have any Stokes vector s on the Poincaré sphere and is produced by a polarization state generator. The Stokes vector of the light scattered by region $a(b)$ is $\mathbf{s}_{a}=M_{a} \mathbf{s}\left(\mathbf{s}_{b}=M_{b} \mathbf{s}\right)$. The light scattered by the scene is analyzed by a polarization state analyzer, which is a generalized polarizer whose eigenstate is the Stokes vector $\mathbf{t}$. In the following, we will denote scalar imaging as the configuration where only one image is measured, Stokes imaging a configuration where the whole Stokes vector is measured (four different analysis states $\mathbf{t}$ are used), and Mueller imaging when the Mueller matrix is measured (four different illumination states $\mathbf{s}$ and four different analysis states $\mathbf{t}$ are used).

Let us first consider scalar imaging. At a given pixel of regions $a$ or $b$, the measured intensity is [ㅁ]

$$
i_{u}=f_{0} t_{o} \times \frac{1}{2} \mathbf{t}^{T} M_{u} \mathbf{s}+n_{u},
$$

where $u=\{a, b\}$ and the superscript $T$ denotes matrix transposition. In this equation, $\mathbf{t}$ and $\mathbf{s}$ are unit intensity Stokes vectors; $f_{0}$ is the illumination flux [in photon(s)]; $t_{o}$ is the time during which the scene is observed; and $n_{u}$, $u=\{a, b\}$, are zero mean Gaussian random variables with variance $\sigma^{2}$. In this Letter, we thus assume that the noise is additive and Gaussian. We also assume that the imaging is photon limited, that is, for any considered value of $t_{o}$, the total measured number of photons does not saturate the capacity of the well of the detector. We consider that the noise can be of two types: with Type I noise, the variance $\sigma^{2}$ is independent of the observation time $t_{o}$, such as readout noise for example. With Type II noise, the variance is proportional to the observation time, that is, $\sigma^{2}=a t_{o}$. This is a pertinent model for dark current noise or shot noise due to unpolarized background illumination.

The additive Gaussian noise model leads to the following expression of the contrast []] : $\mathcal{C}(\mathbf{s}, \mathbf{t})=\left(i_{a}-i_{b}\right)^{2} / \sigma^{2}$. Using Eq. (1), this contrast can also be written as

$$
\mathcal{C}(\mathbf{s}, \mathbf{t})=\left(\frac{f_{0} t_{o}}{\sigma}\right)^{2} \times \frac{1}{4}\left(\mathbf{t}^{T} \Delta M \mathbf{s}\right)^{2},
$$

where $\Delta M=M_{a}-M_{b}$ is the difference between the Mueller matrices of the two regions. The contrast in Eq. (2) is a function of both the illumination and the analysis state. In practice, one will determine the vectors $\mathbf{s}$ and $\mathbf{t}$ that maximize it, and the maximal achievable contrast is $\mathcal{C}_{\text {scalar }}=\max _{\mathrm{s}, \mathrm{t}}[\mathcal{C}(\mathbf{s}, \mathbf{t})]$. An efficient way of determining this maximum has been proposed in [4].

Let us now consider Stokes imaging systems. Let us define the matrix $W$ whose rows are the Stokes vectors of the four analysis states $\mathbf{t}_{i}^{T}, i \in\{1,4\}$, where $\mathbf{t}_{i}$ are unit intensity, fully polarized Stokes vectors that are usually chosen so as to minimize the variance of the estimated Stokes vector [5]. The four measured intensities can be stacked in a four-dimensional vector:

$$
\mathbf{i}_{u}=\frac{f_{0} t_{o}}{4} \times \frac{1}{2} W M_{u} \mathbf{s}+\mathbf{n}_{u},
$$

where $u=\{a, b\}$; $\mathbf{s}$ is the Stokes vector of the illumination; and the vector $\mathbf{n}_{u}, u=\{a, b\}$, is a realization of a 
four-dimensional white Gaussian random vector $\mathbf{n}$ with variance $\sigma^{2}$. Its covariance matrix is $\Gamma_{n}=\sigma^{2} \mathcal{I}_{4}$, where $\mathcal{I}_{4}$ is the $4 \times 4$ identity matrix. The factor $f_{0} t_{0} / 4$ comes from the fact that the observation time $t_{o}$ is constant, so that if four intensity measurements are performed during this time, the integration time for each measurement is equal to $t_{o} / 4$. The adequate expression of the contrast is [3] $\mathcal{C}(\mathbf{s})=\left(\mathbf{i}_{a}-\mathbf{i}_{b}\right)^{T} \Gamma_{n}^{-1}\left(\mathbf{i}_{a}-\mathbf{i}_{b}\right)$. Taking into account Eq. (3), it can also be written as

$$
\mathcal{C}(\mathbf{s})=\left(\frac{f_{0} t_{o}}{\sigma}\right)^{2}\left(\frac{1}{4}\right)^{2} \times \frac{1}{4}\|(W \Delta M) \mathbf{s}\|^{2},
$$

where $\|$.$\| stands for the Euclidian norm on four-$ dimensional vectors. It is a function of the illumination state. In practice, one will determine the vector $\mathbf{s}$ that maximizes it [3], and the maximal achievable contrast is $\mathcal{C}_{\text {Stokes }}=\max _{\mathbf{s}}[\mathcal{C}(\mathbf{s})]$.

Let us now consider Mueller imaging systems. In this case, one uses four different illumination Stokes vectors $\mathbf{s}_{i}, i \in[1,4]$, that form the columns of a matrix $X$. One also has four analysis states in the matrix $W$ defined above. One measures 16 intensities that can be gathered in the following matrices:

$$
I_{u}=\frac{f_{0} t_{o}}{16} \times \frac{1}{2} W M_{u} X+N_{u},
$$

where $u=\{a, b\}$ and $N_{u}$ are $4 \times 4$ random matrices whose elements are independent Gaussian random variables with variance $\sigma^{2}$. The factor $f_{0} t_{o} / 16$ stands for the fact that 16 intensity measurements are performed during the observation time $t_{0}$. The contrast can be written as

$$
\mathcal{C}=\left(\frac{f_{0} t_{o}}{\sigma}\right)^{2}\left(\frac{1}{16}\right)^{2} \times \frac{1}{4}\|W \Delta M X\|_{f}^{2},
$$

where $\|Q\|_{f}=\left[\sum_{i j} Q_{i j}^{2}\right]^{1 / 2}$ denotes the Frobenius norm of a matrix $Q$.

Using Eqs. (2), (4), and (6), the expressions of the contrast in the three above-mentioned imaging modalities can be written in the following way:

$$
\begin{aligned}
\mathcal{C}_{\text {Scalar }}^{1} & =\frac{f_{0}^{2} t_{o}^{2}}{4 \sigma^{2}} \times \max _{\mathbf{s}, \mathbf{t}}\left[\left(\mathbf{t}^{T} \Delta M \mathbf{s}\right)^{2}\right], \\
\mathcal{C}_{\text {Stokes }}^{1} & =\frac{1}{4}\left[\frac{f_{0}^{2} t_{o}^{2}}{4 \sigma^{2}}\right] \times \max _{\mathbf{s}}\left[\frac{1}{4} \sum_{k=0}^{3}\left(\mathbf{t}_{k}^{T} \Delta M \mathbf{s}\right)^{2}\right], \\
\mathcal{C}_{\text {Mueller }}^{1} & =\frac{1}{16}\left[\frac{f_{0}^{2} t_{o}^{2}}{4 \sigma^{2}}\right] \times\left[\frac{1}{16} \sum_{k=0}^{3} \sum_{l=0}^{3}\left(\mathbf{t}_{k}^{T} \Delta M \mathbf{s}_{l}\right)^{2}\right] .
\end{aligned}
$$

Please note that in the expressions of $\mathcal{C}_{\text {Stokes }}^{1}$ and $\mathcal{C}_{\text {Mueller }}^{1}$, we have split the factor $(1 / N)^{2}(N$ being the number of measurements) that appears in Eqs. (4) and (ㅁ) into two parts: one factor $1 / N$ in front of the first term and another in front of the second term. This second term thus appears as a sum of $N$ terms divided by $N$. The expressions in Eq. (7) make it possible to compare the maximum contrast achievable by the three modalities. The first term of the right-hand side represents the intensity signal-to-noise ratio, $\mathrm{SNR}=f_{0}^{2} t_{o}^{2} / 4 \sigma^{2}$, divided by the number of measurements ( 1 for scalar imaging, 4 for Stokes imaging, and 16 for Mueller imaging). The value of this term strongly depends on the type of noise. Indeed, for noise of Type I, the variance is independent of the measurement duration and the contrast thus decreases proportionally to the number of performed measurements. This is easily understood because each measurement adds up a constant amount of noise, thus reducing the contrast. On the other hand, for noise of Type II, the variance is proportional to the integration time and can thus be written $\sigma^{2}=a t_{o} / N$, where $N$ is the number of measurements. In this case, the first term in Eq. (7) is equal to $f_{0}^{2} t_{o} / a$ for the three imaging modes. In the presence of Type II noise, the contrasts can thus be written as

$$
\begin{aligned}
\mathcal{C}_{\text {Scalar }}^{2} & =\frac{f_{0}^{2} t_{o}}{4 \alpha} \times \max _{\mathbf{s}, \mathbf{t}}\left[\left(\mathbf{t}^{T} \Delta M \mathbf{s}\right)^{2},\right. \\
\mathcal{C}_{\text {Stokes }}^{2} & =\frac{f_{0}^{2} t_{o}}{4 \alpha} \times \max _{\mathbf{s}}\left[\frac{1}{4} \sum_{k=0}^{3}\left(\mathbf{t}_{k}^{T} \Delta M \mathbf{s}\right)^{2}\right], \\
\mathcal{C}_{\text {Mueller }}^{2} & =\frac{f_{0}^{2} t_{o}}{4 \alpha} \times\left[\frac{1}{16} \sum_{k=0}^{3} \sum_{l=0}^{3}\left(\mathbf{t}_{k}^{T} \Delta M \mathbf{s}_{l}\right)^{2}\right] .
\end{aligned}
$$

One can note that if the two types of noises are simultaneously present, the total variance can be written as $\sigma^{2}=\sigma_{0}^{2}+a t_{0} / N$, and the situation is intermediary between Eqs. (7) and (8).

The second term of the right-hand sides of Eqs. (7) and (8) is a sum of $N$ positive terms divided by $N$. Obviously, $\overline{\mathcal{C}}_{\text {Scalar }}^{i} \geq \mathcal{C}_{\text {Stokes }}^{i} \geq \mathcal{C}_{\text {Mueller }}^{i}$, where $i \in\{1,2\}$, stands for the type of noise. Indeed, $\mathcal{C}_{\text {Stokes }}^{i}$ can be seen as a version of $\mathcal{C}_{\text {Mueller }}^{i}$, where the illumination state of polarization has been optimized, and $\mathcal{C}_{\text {Scalar }}^{i}$ a version where both the illumination and the analysis states have been optimized. Consequently, scalar imaging is the most efficient approach, and the architectures that acquire more than one image will lead to a lower contrast. As a side effect

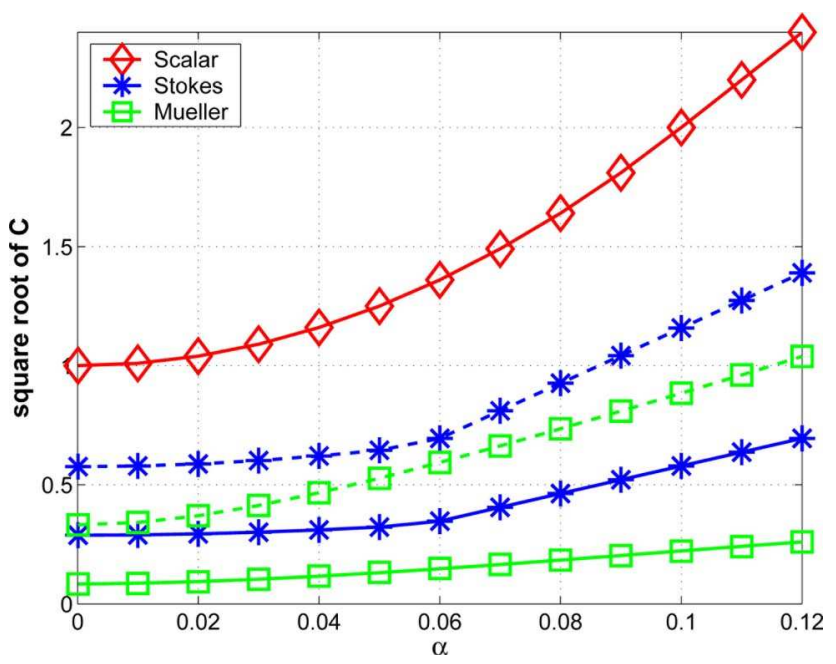

Fig. 1. (Color online) Normalized values of the square root of the contrast for scalar, Stokes, and Mueller images as a function of $\alpha$; see Eq. (9). $\oslash,\left[\mathcal{C}_{\text {Scal }}^{i}(\alpha) / \mathcal{C}_{\text {Scal }}^{i}(0)\right]^{1 / 2} ; *,\left[\mathcal{C}_{\text {Stokes }}^{i}(\alpha) / \mathcal{C}_{\text {Scal }}^{i}(0)\right]^{1 / 2}$; $\square,\left[\mathcal{C}_{\text {Mueller }}^{i}(\alpha) / \mathcal{C}_{\text {Scal }}^{i}(0)\right]^{1 / 2}$. In each case: solid line, $i=1$ (Type I noise); dotted line, $i=2$ (Type II noise). 


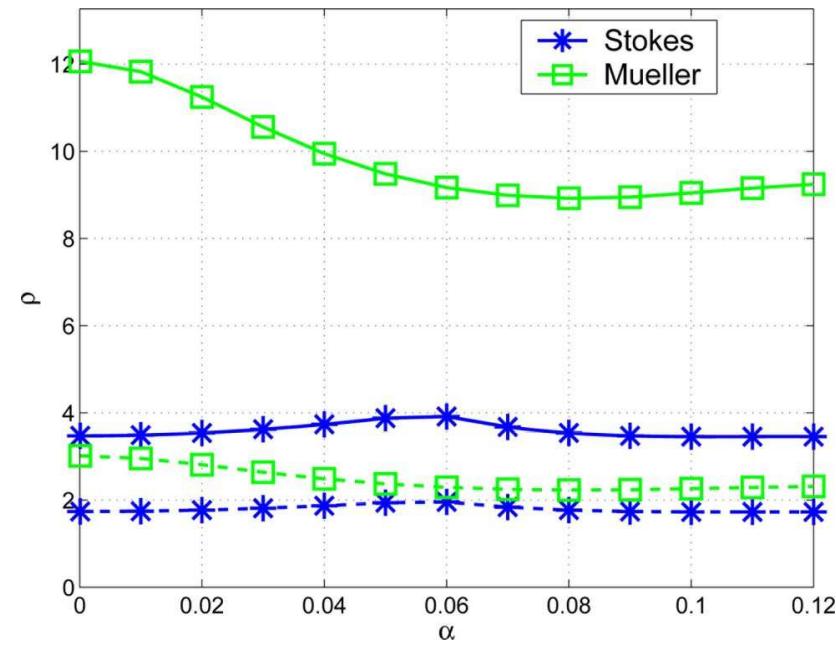

Fig. 2. (Color online) Gain of scalar imaging with respect to Stokes and Mueller imaging as a function of $\alpha$; see Eq. (9).*, $\rho_{\text {Stokes }}^{i}(\alpha) ; \square, \rho_{\text {Mueller }}^{i}(\alpha)$. In each case: solid line, $i=1$ (Type I noise); dotted line, $i=2$ (Type II noise).

of the present study, one can notice that the value $\mathcal{C}_{\text {Scalar }}^{i}$ can be used as a pertinent metric for quantifying the intrinsic difficulty of a detection task with polarization imaging.

As anillustrative example, let us assume that region $a$ has the following Mueller matrix:

$$
M_{a}(\alpha)=\left[\begin{array}{cccc}
0.8 & \alpha & 0 & 0 \\
\alpha & 0.5 & 0 & 0 \\
0 & 0 & 0.5 & 0 \\
0 & 0 & 0 & 0.4
\end{array}\right]
$$

where $\alpha$ is a parameter that can vary between 0 and 0.12 . Let us also assume that the background region has the following diagonal Mueller matrix: $M_{b}=$ diag $(0.8,0.5,0.5,0.3)$. For Stokes imaging, we have chosen the matrix $W$ implemented by the optimal rotatable retarder-fixed polarizer polarimeter described in [5], and for Mueller imaging, we have chosen $X=W^{T}$. We have plotted in Fig. 1, as a function of $\alpha$, the square roots of the values of the contrast obtained with the three architectures, normalized in the following way: $\left[\mathcal{C}_{\text {Scal }}^{i}(\alpha) / \mathcal{C}_{\text {Scal }}^{i}(0)\right]^{1 / 2}$, $\left[\mathcal{C}_{\text {Stokes }}^{i}(\alpha) / \mathcal{C}_{\text {Scal }}^{i}(0)\right]^{1 / 2}$, and $\left[\mathcal{C}_{\text {Mueller }}^{i}(\alpha) / \mathcal{C}_{\text {Scal }}^{i}(0)\right]^{1 / 2}$, where $i \in\{1,2\}$.Indeed,for Stokes and Mueller imaging systems, there are two curves in the graph: the solid curves correspond to noise of Type I [Eq. (7)], and the dotted curves to noise of Type II [Eq. (8)]. Note that because of this normalization, the curves for Type I and Type II noises overlap in the scalar case.
It is seen that for all imaging modes, the contrast increases with $\alpha$. This is understandable because $\alpha$ corresponds to the polarizance/diattenuation factor of $M_{a}(\alpha)$ : when it increases, the difference between $M_{a}(\alpha)$ and $M_{b}$ increases. It is also verified that the scalar imaging mode provides the highest value of contrast. To have a better idea of the gain in contrast obtained by using scalar imaging, we have plotted in Fig. 2 the following ratios: $\rho_{\text {Stokes }}^{i}(\alpha)=\left[\mathcal{C}_{\text {Scalar }}^{i}(\alpha) / \mathcal{C}_{\text {Stokes }}^{i}(\alpha)\right]^{1 / \overline{2}}$ and $\rho_{\text {Mueller }}^{i}(\alpha)=$ $\left[\mathcal{C}_{\text {Scalar }}^{i}(\alpha) / \mathcal{C}_{\text {Mueller }}^{i}(\alpha)\right]^{1 / 2}$, with $i \in\{1,2\}$. In the presence of Type I noise, the contrast obtained with scalar imaging is about ten times higher than with Mueller imaging and four times higher than with Stokes imaging. For Type II noise, these ratios are smaller but still around 2. Of course, the values of these ratios depend on the matrices $M_{a}$ and $M_{b}$, but the ranking between the three imaging modalities will always be the same.

In conclusion, we have shown that in the presence of additive noise, simple scalar imaging with optimized illumination and analysis states always yields better contrast than Stokes and Mueller imaging. This result provides an important element in the choice of polarimetric imaging architectures. Of course, this conclusion is valid when the only concern is to optimize the contrast between two regions with definite polarimetric signatures. When discriminating multiple targets, or if the objective is to have insight into the physics of the scene, multidimensional polarimetric measurements may be needed, and strategies based on partial estimation of the Mueller matrix may be more appropriate [6]. As a perspective, it can be noted that noise models other than the additive one may be more relevant in some situations, such as signaldependent shot noise or speckle noise. In such cases, the relevant expressions of the contrast are different and so may be the conclusions in terms of the influence of the number of measurements.

\section{References}

1. A. A. Swartz, H. A. Yueh, J. A. Kong, L. M. Novak, and R. T. Shin, J. Geophys. Res. 93, 15252 (1988).

2. M. Floc'h, G. Le Brun, C. Kieleck, J. Cariou, and J. Lotrian, Pure Appl. Opt. 7, 1327 (1998).

3. F. Goudail, Opt. Lett. 34, 121 (2009).

4. F. Goudail and A. Bénière, Opt. Lett. 34, 1471 (2009).

5. D. S. Sabatke, M. R. Descour, E. L. Dereniak, W. C. Sweatt, S. A. Kemme, and G. S. Phipps, Opt. Lett. 25, 802 (2000).

6. J. S. Tyo, Z. Wang, S. J. Johnson, and B. Hoover, Appl. Opt. 49, 2326 (2010). 